\title{
High Density Intramural Mapping of Post-Infarct Premature Ventricular Contractions.
}

\author{
Eugene Downar ${ }^{1}$, Michiel Janse ${ }^{2}$, Abhishek Bhaskaran ${ }^{1}$, Ahmed Niri ${ }^{1}$, Arulalan \\ Velluppillai $^{1}$, Stéphane Massé ${ }^{1}$, and Kumaraswamy Nanthakumar ${ }^{3}$ \\ ${ }^{1}$ University Health Network \\ ${ }^{2}$ Academic Medical Center \\ ${ }^{3}$ Toronto General Hospital
}

January 17, 2021

\begin{abstract}
Background: Spontaneous ventricular premature contractions (PVCs) in the post infarct milieu is assumed to be due to automaticity. However, the mechanism has not been studied with appropriate mapping tools. Objective: To study the mechanism of spontaneous PVCs with high density intramural mapping in a canine model, to test the hypothesis that postinfarct PVCs are due to re-entry rather than automaticity. Methods: In 15 anesthetized dogs, using 768 intramural unipolar electrograms, simultaneous recordings were made. After $30 \mathrm{mins}$ of stabilization, recordings were made during the first 10 minutes of ischemia, and activation maps were constructed of individual beats. Acute ischemia was produced by clamping the left anterior descending coronary artery proximal to the first diagonal branch. The analysis was limited to the activation pattern of spontaneous ventricular beats. Results: In all experiments ST-T alternans occurred. In 8 of 15 dogs spontaneous ventricular beats occurred. In all 8 of these experiment earliest, ectopic activity occurred in the endocardium, well within the ischemic zone. From there, activity spread rapidly along the subendocardium, with endo-to epicardial spread along the non ischemic myocardium. Epicardial breakthrough always occurred at the border of the ischemic myocardium. In 3 dogs, delayed potentials were observed, which were earliest at the ischemic epicardium and extended transmurally with increasing delay towards the endocardium, where they culminated in a premature beat. Conclusion: Graded responses that occur with each sinus beat intramurally, when able to propagate from epicardium to endocardium is the mechanism by which PVCs are generated in post-infarct myocardium.
\end{abstract}

High Density Intramural Mapping of Post-Infarct Premature Ventricular Contractions.

Eugene Downar, $\mathrm{MD}^{*}$; Michiel J Janse, $\mathrm{MD}, \mathrm{PhD}^{+}$; Abhishek Bhaskaran MD, $\mathrm{PhD}^{*}$;

Ahmed Niri*, Arulalan Velluppillai*, Stéphane Massé MASc*;

Kumaraswamy Nanthakumar MD*, FRCPc.

Address Correspondence to:

Kumaraswamy Nanthakumar, MD, FRCPC

Division of Cardiology, University Health Network

Toronto General Hospital, 150 Gerrard Street West, GW3-526

Toronto, Ontario, Canada, M5G 2C4

$\mathrm{Ph}:(416) 340-4442$ 
Fax: (416) 340-4457

kumar.nanthakumar@uhn.ca

* Division of Cardiology. Toronto General Hospital, University Health Network. Toronto, Ontario, Canada.

+ Experimental and Molecular Cardiology Group, Academic Medical Center, Amsterdam, Netherlands.

Conflicts of Interest: None

Acknowledgment: This work was supported by the Heart and Stroke Foundation of Ontario.

\section{Abstract}

Background: Spontaneous ventricular premature contractions (PVCs) in the post infarct milieu is assumed to be due to automaticity. However, the mechanism has not been studied with appropriate mapping tools.

Objective: To study the mechanism of spontaneous PVCs with high density intramural mapping in a canine model, to test the hypothesis that post-infarct PVCs are due to re-entry rather than automaticity.

Methods: In 15 anesthetized dogs, using 768 intramural unipolar electrograms, simultaneous recordings were made. After 30 mins of stabilization, recordings were made during the first 10 minutes of ischemia, and activation maps were constructed of individual beats. Acute ischemia was produced by clamping the left anterior descending coronary artery proximal to the first diagonal branch. The analysis was limited to the activation pattern of spontaneous ventricular beats.

Results: In all experiments ST-T alternans occurred. In 8 of 15 dogs spontaneous ventricular beats occurred. In all 8 of these experiment earliest, ectopic activity occurred in the endocardium, well within the ischemic zone. From there, activity spread rapidly along the subendocardium, with endo-to epicardial spread along the non ischemic myocardium. Epicardial breakthrough always occurred at the border of the ischemic myocardium. In 3 dogs, delayed potentials were observed, which were earliest at the ischemic epicardium and extended transmurally with increasing delay towards the endocardium, where they culminated in a premature beat.

Conclusion: Graded responses that occur with each sinus beat intramurally, when able to propagate from epicardium to endocardium is the mechanism by which PVCs are generated in post-infarct myocardium.

\section{Introduction}

The inability to map the intramural space in humans has led to tacit acceptance of using either endocardial or epicardial data to determine targets for ablation and or deciding mechanism of post infarct Premature Ventricular Contraction (PVC). Though the significance of the intramural space in ventricular arrhythmogenesis has recently been highlighted by us and others (1-4), it has not been studied with high resolution mapping in post-infarct PVCs.

Additionally, the prevailing theory of post-infarct PVC has been attributed to purkinje depolarizations in the border zones (5) as the driver, setting up automaticity as dogma. However, if upstream mechanisms are not adequately evaluated, mapping data could easily be misinterpreted based on downstream endo/ epi cardial mapping findings. Potential mechanisms such as depolarization of non-ischemic myocardium close to the ischemic border by injury currents (6), or transmural re-entry have not been studied in detail. As such we devised a high density mapping scheme with 768 simultaneous intramural electrograms. As ST-T alternans and injury currents are best monitored by unipolar electrograms we elected to use an exclusive unipolar scheme developed by Downar to evaluate mechanism of spontaneous post-infarct PVCs (7-8).

\section{Methods}

Studies conformed to the guiding principles of the Declaration of Helsinki. In 15 dogs (14-25 kg) anesthesia was induced and maintained by Nembutal $30 \mathrm{mg} / \mathrm{kg}$. Positive pressure ventilation was maintained through an endocardial tube to maintain normal blood gases. The chest was opened through a medial sternotomy. 
The left anterior descending coronary artery was dissected free along a $5 \mathrm{~mm}$ segment proximal to the first diagonal branch. The left anterior descending coronary artery was abruptly occluded with a hemoclip for a period of 15 minutes. 768 unipolar electrodes on 112 transmural multipolar needle electrodes were inserted into the heart in a standardized array illustrated in Figure 1. Care was taken to avoid epicardial vessels. Each needle was fabricated from 23 gauge steel with either 4 or 8 tungsten wire electrodes set in epoxy at 1, 2 or 4 $\mathrm{mm}$ intervals (8). This array provided a total of 768 unipolar electrograms with a reference electrode placed adjacent to the left first costosternal junction. A 20 to 30 minute control period was allowed after insertion of the last needle for injury currents to subside. At the conclusion of each experiment the heart was excised with the electrodes in place and the relative relationship of the needles confirmed. Each of the 768 channels was sampled with a frequency of 960 samples/sec and filtered to provide a bandwidth of 0.07-170 HZ (7). Local activation time was defined as dominant negative deflection in the unipolar electrogram, an automated algorithm that determines slope of $-5 \mathrm{~V} / \mathrm{sec}$ was used to annotate detection in similar electrograms in previous work $(7,8)$ We focused our analysis on the spontaneous PVCs that triggered ventricular arrhythmias.

\section{Results}

Typical ST T sequence of changes in unipolar electrograms recorded from the ischemic myocardium is shown in Figure 1B. These recordings were obtained from an electrode $2 \mathrm{~mm}$ below the epicardial surface on needle D10 of the standardized array in figure 1A. Within 90 seconds of occlusion ischemic changes were well developed and by 3 minutes alternans in the ST-T segment become very marked. Such alternans occurred in all of the 15 hearts we studied.

Of the fifteen hearts we studied only five developed ventricular tachycardias that degenerated into ventricular fibrillation. In those animals that did have arrhythmias, the alternans described further evolved into more complex cycles in which each morphology of the electrogram was repeated every 3-7 sinus beats as illustrated in Figure 2A. In these 5 hearts, the last beat of each sequence was usually followed by a spontaneous premature ventricular beat. In 3 of the 5 experiments with arrhythmias the alternans evolved to produce increasingly delayed intermittent potentials of varying magnitude which extended into diastole (Figure 2B). These delayed potentials were at their earliest at the epicardium and extended transmurally with increasing delay towards the endocardium. When maximally expressed, as shown in Figure 2B, they culminated in a ventricular premature beat.

The earliest site of activation of PVC was always found in the sub-endocardium overlying the ischemic region, well away from the border region Figure 3. The epicardial breakthrough always occurred at the border of the ischemic region with separate broad wave fronts sometimes appearing in diametrically opposite locations as shown in Figure 3.

\section{Discussion}

We have demonstrated with high density intramural mapping of post-infarct PVC, that transmural re-entry with graded responses that propagate in from epicardium to endocardium is the mechanism of PVC in our experimental model. Even though transmural re-entry has been demonstrated before, the graded response in myocardium from epicardium to endocardium during sinus rhythm culminating in PVC is a novel finding. In agreement with the results of the present study, earlier studies also showed that ectopic activity during acute ischemia originated in the sub-endocardium. $(8,10,11)$ A role for the Purkinje system was suggested based on the argument that whenever sub-endocardial recordings showed Purkinje activity, the Purkinje spikes preceded myocardial deflections during premature beats and subsequent beats of a tachycardi and when hearts were paced via the electrode terminal from which Purkinje activity had been recorded, the epicardial activation pattern was the same as during the spontaneous premature beat. Janse et al, probably because the number of electrodes from which electrograms could be recorded simultaneously was too small $(\mathrm{n}=60)$, no evidence for transmural re-entry was found. (11)

Reentry within the intramural space has not been studied in ischemic PVCs for inability map the intramural space. The sampling rate needed to appropriately map Purkinje as detailed by Ideker (13) effectively sets up a conundrum for high resolution mapping of the intramural space as it is not possible to have such high 
sampling rate and adequate number of mapping channels.

In the present study, no Purkinje activity was recorded. This was most likely due to the sampling rate used (960 samples/sec). Janse et al could only record Purkinje activity when the sampling rate was raised to once per $0.5 \mathrm{~ms}$ and when the amplification factor was increased.(11) This could only be obtained at the cost of less electrodes from which activity could simultaneously be recorded. The advantage of the present study lies in the number of simultaneously recorded electrograms (768) so that the mechanism of intramural re entry could be detected. The disadvantage is the sampling rate precludes recording of Purkinje activity. Although the origin of PVC was within the ischemic sub endocardium, epicardial breakthrough occurred at the ischemic border, often at more than one separate front. Epicardial mapping alone would have erroneously indicated the border zone as the source.Transmural re-entry has been observed in different settings. In canine hearts with an evolving myocardial infarction, recordings from 232 cardiac sites revealed intramural re entry (14). In human hearts with a chronic myocardial infarction, transmural re entry was documented, (15) although in the latter study focal mechanisms were identified as well.

\section{Conclusion}

Transmural graded responses from epicardium to endocardium during sinus beats in the peri-ischemic region that reenters adjacent healthy regions is the mechanism of post infarct PVC formation.

\section{References}

1. Bhaskaran A, Nayyar S, Porta-Sanchez A, et al. Direct and indirect mapping of intramural space in ventricular tachycardia. Heart Rhythm 2020; 17, 439-436

2. Nair K, Umapathy K, Farid T, et al. Intramural Activation During Early Human Venticular Fibrillation. Circulation-Arrhythmia and Electrophysiology 2011; 4, 692-703

3. Jackson N, Masse S, Zamiri N, et al. Mechanisms of Long-Duraion Ventricular Fibrillation in Human Hearts and Experimental Validation in Canine Purkinje Fibers. Journal of Amercan College of Cardioogy EP: Clinical Electrophysiology 2015; 1(3), 187-197

4. Tung R, Raiman M, Liao H, et a. Simultaneous Endocardial and Epicardial Delineation of 3D Reentrant Ventricular Tachycrdia. Journal of the American College of Cardiology 2020; 75(8) 884-897

5. Haissaguerre M, Shah DC, Jais P, et al. Role of Purkinje conduction system in triggering of idiopathic ventricular fibrillation. The lancet 2002; 359,677-678

6. Sarrazin JF, Good E, Kuhne M, et al. Mapping and ablation of frequent post-infarction premature ventricular complexes. Journal of Cardiovascular Electrophysiology 2010; 21(9), 1002-1008

7. Parson I, Mendler P, Downar E. On-line mapping: an analog approach using video and multiplexing techniques. American Journal of Physiology-Heart and Circulatory Physiology 1982; 242(4), H526-H35

8. Downar E, Janse MJ, Durrer D. The effect of acute coronary artery occlusion on subepicardial transmembrane potentials in the intact porcine heart. Circulation 1977; 56, 217-224

9. Patterson E, Kalcich M, Scherlag BJ. Phase 1B ventricular arrhythmia in the dog: localized reentry within the mid-myocardium. Journal of interventional cardiac electrophysiology 1998;2(2):145-161.

10. Kleber AG, Janse MJ, Capelle FJ, Durrer D. Mechanism and time course of ST and TQ segment changes during acute regional myocardial ischemia in the pig heart determined by extracellular and intracellular recordings. Circulation Research 1978; 42(5), 603-613

11. Janse MJ, van Capelle FJ. Electrotonic interactions across an inexcitable region as a cause of ectopic activity in acute regional myocardial ischemia. A study in intact porcine and canine hearts and computer models. Circulation Research 1982; 50(4),527-537.

12. Bogun F, Crawford T, Chalfoun N, et al. Relationship of frequent postinfarction premature ventricular complexes to the reentry circuit of scar-related ventricular tachycardia. Heart Rhythm 2008; Mar, $5(3): 367-374$.

13. Huang J, Dosdall D J, Cheng KA, et al. The importance of Purkinje activation in long duration ventricular fibrillation. Journal of the American Heart Association 2014; 3(1), e000495

14. Takahashi T, van Dessel P, Lopshire JC, et al. Optical mapping of the functional reentrant circuit of ventricular tachycardia in acute myocardial infarction. Heart Rhythm 2004;1(4):451-459. 
15. De Bakker J, Van Capelle F, Janse MJ, et al. Reentry as a cause of ventricular tachycardia in patients with chronic ischemic heart disease: electrophysiologic and anatomic correlation. Circulation 1988;77(3):589-606.

\section{Figure Legend}

Figure 1(a) Intramural electrode array is depicted on the polar projection of canine heart. Shaded area denotes the boundary of ischemic region. 1(b) Sub epicardial electrograms from the ischemic zone from three separate experiments between 3 and 5 minutes after coronary artery occlusion. PVC (indicated by arrows) was in each case preceded by an augmentive cycle of ST-T wave alternans, which was repetitive.

Figure 2 Transmural unipolar electrograms from needle in the center of ischemic region recorded at $2 \mathrm{~mm}$ intervals from the epicardium, showing a continuous tracing displayed during the ST-T wave alternans 4'30" after coronary occlusion. The arrows indicate the development of transmural graded responses with alternate sinus beats, culminating in the PVC.

Figure 3 Activation map of PVC. The earliest activation occurred at the ischemic sub-endocardium. Spread of activation in the sub-endocardium is relatively rapid progressing in a radial fashion across the subendocardium overlying the ischemic region. Sub-epicardial activation emerged later at the ischemic border in two separate locations diametrically opposite across the ischemic region. Spread into the ischemic subepicardium progressed slowly, persisting up to $180 \mathrm{~ms}$ after the earliest onset of PVC, however failing to activate all of the ischemic region (white area).

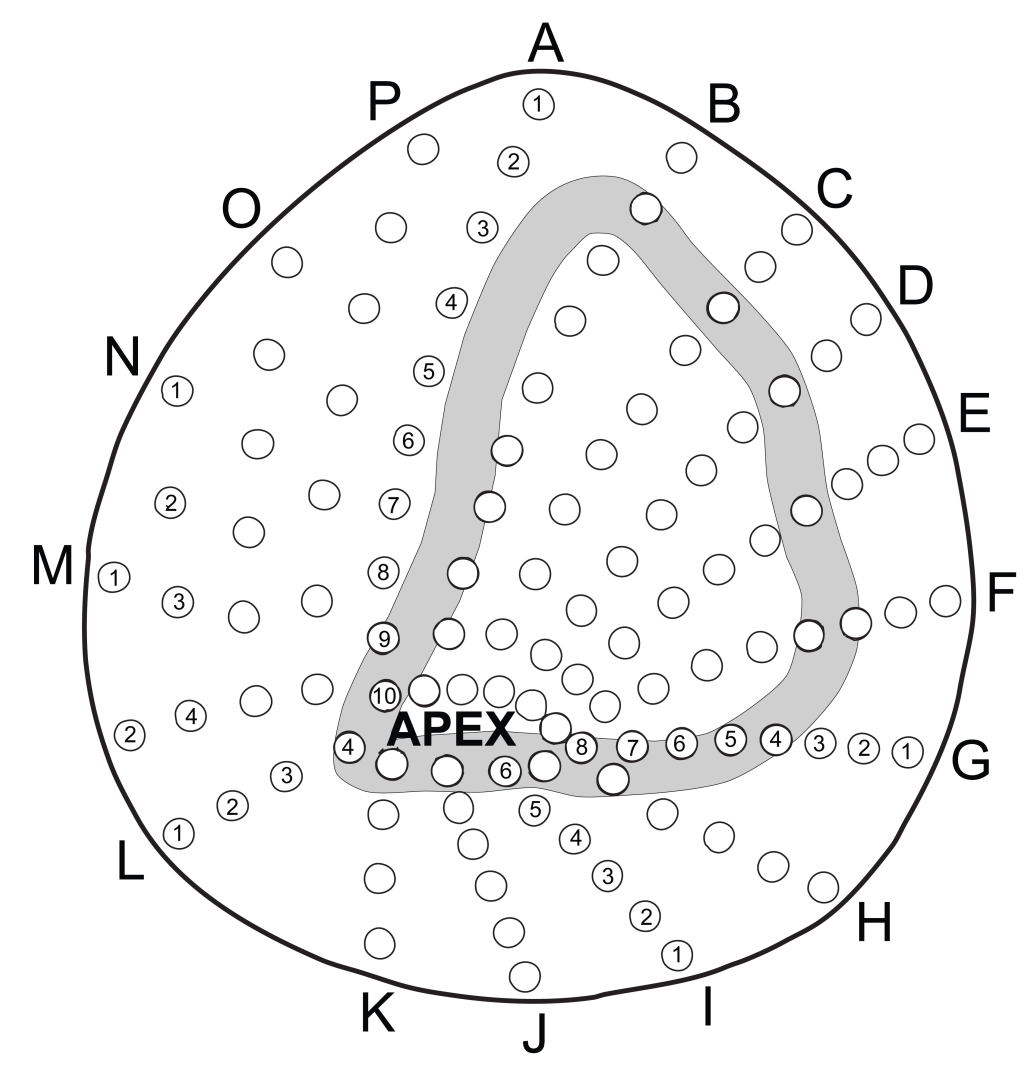



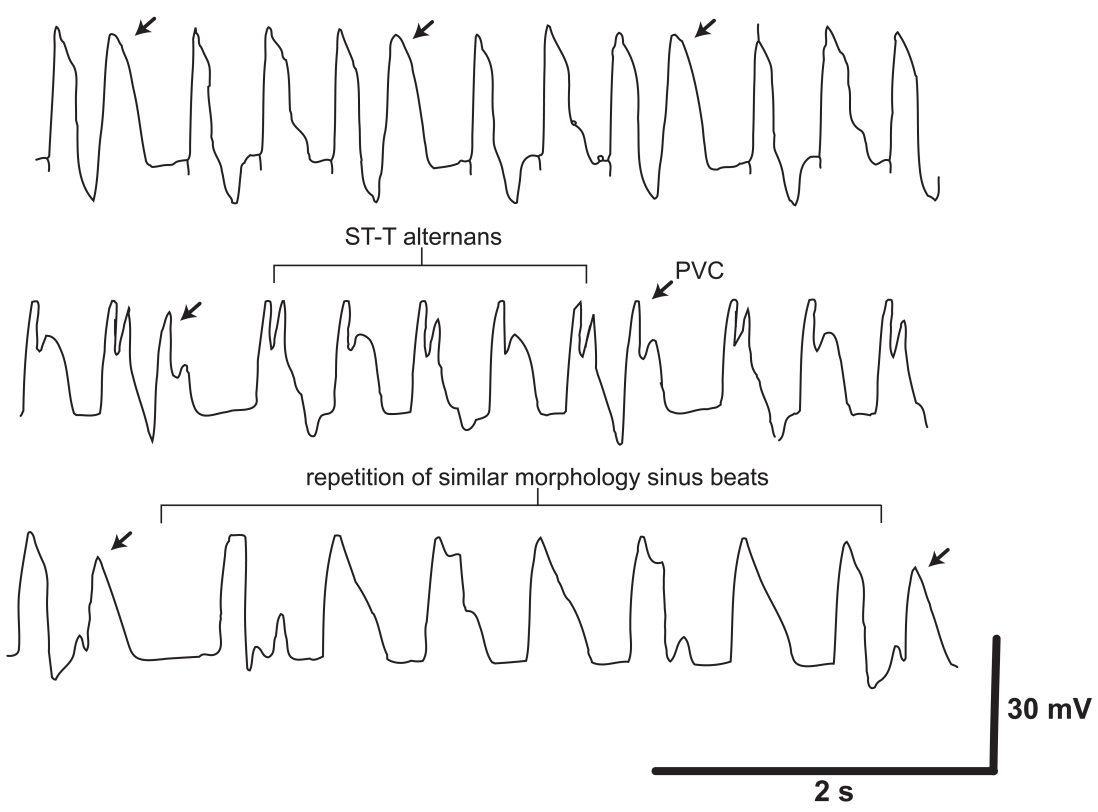

\section{Epicardium}

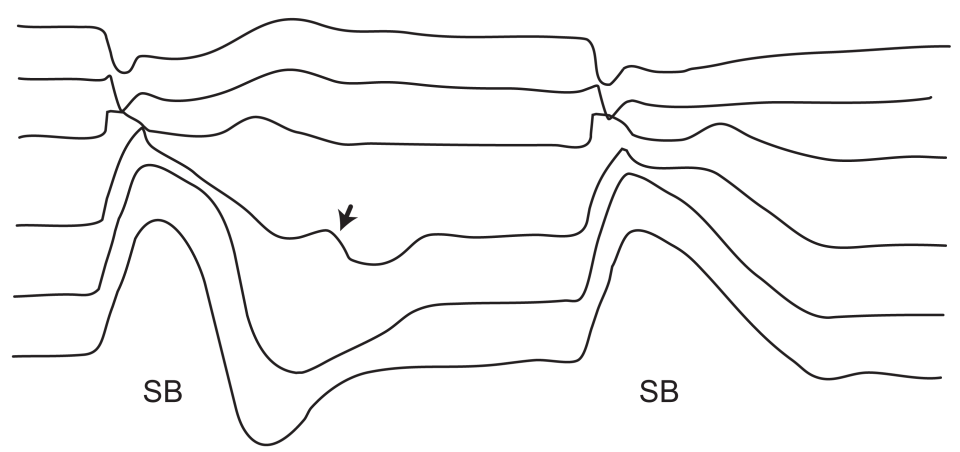

Transmural graded response terminating in a PVC

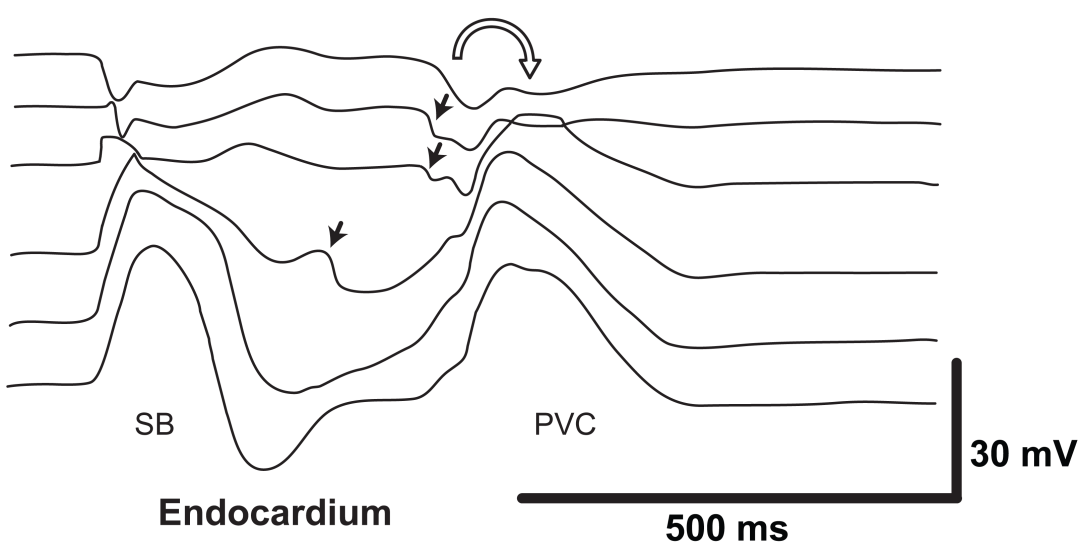




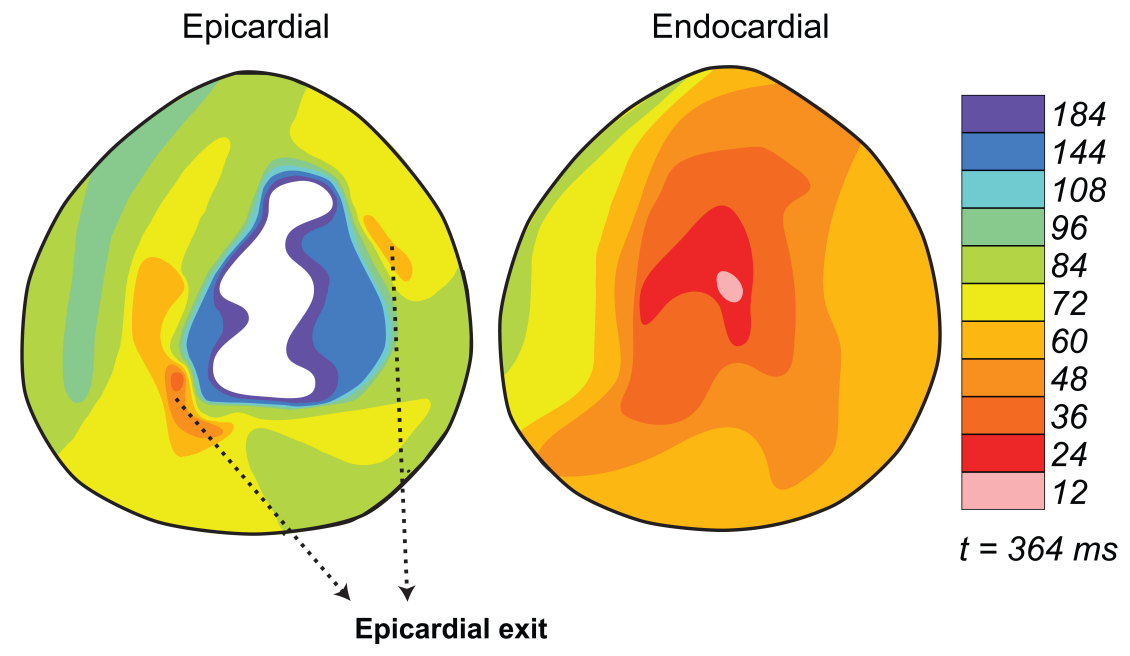

